\title{
pharma-kritik
}

AZB 9500 Wil

ISSN 1010-5409

24. Mai 2006

Jahrgang 27

Nummer 14/2005

\section{Nebenwirkungen aktuell}

\section{ROSIGLITAZON}

Rosiglitazon, ein «Insulinsensitizer», kann bei Typ-2Diabetes als Monotherapie oder in Kombination mit anderen Antidiabetika verwendet werden. Über Probleme der Glitazone haben wir bereits vor rund vier Jahren einmal berichtet.

Übersichten zu Rosiglitazon:

Koch T, Masche UP. pharma-kritik 1999; 21: 33-4

Wagstaff AJ, Goa KL. Drugs 2002; 62: 1805-37

Markenname: Rosiglitazon $=$ Avandia $^{\circledR}$

\section{Makulaödem}

Ein 55-jähriger Mann beobachtete über den Zeitraum von zwei Wochen eine allmähliche Abnahme der Sehkraft beider Augen. Dieser Patient hatte einen seit vielen Jahren bestehenden Typ-2-Diabetes mit verschiedenen Komplikationen (Retinopathie, Neuropathie, Nephropathie). Er wurde mit folgenden Medikamenten behandelt: Insulin-Glargin (Lantus ${ }^{\circledR}$ ), Rosiglitazon, Atorvastatin (Sortis ${ }^{\circledR}$ ), Amlodipin (Norvasc ${ }^{\circledR}$ u.a.), Quinapril (Accupro ${ }^{\circledR}$ u.a.), Hydrochlorothiazid (Esidrex ${ }^{\circledR}$ ) und Sertralin (Gladem $^{\circledR}$, Zoloft ${ }^{\circledR}$ u.a.). Ein Monat vor dem Auftreten der Sehstörung war die Rosiglitazon-Dosis von $2 \mathrm{mg}$ auf $8 \mathrm{mg}$ täglich erhöht worden. Gleichzeitig mit der Sehstörung traten auch periphere Ödeme auf. Ophthalmoskopisch liess sich ein Makulaödem nachweisen. Die Rosaglitazon-Dosis wurde wieder auf $2 \mathrm{mg}$ täglich reduziert, worauf das Makulaödem innerhalb von 3 Wochen verschwand. Auch die Sehkraft normalisierte sich wieder.

Colucciello M. Arch Ophthalmol 2005; 123: 1273-5

Etwa 15\% der Personen, die an einem Typ-2-Diabetes leiden und mit Insulin behandelt werden, haben ein Ödem der Makula. Verschwommenes Sehen, reduzierte Farbempfindlichkeit und reduzierte Dunkeladaptation sind die wichtigsten Symptome.

Die kanadischen Gesundheitsbehörden haben Kenntnis von 9 Personen, die im Zusammenhang mit der Einnahme von Rosiglitazon eine Beeinträchtigung des Visus erlitten. Jedoch nur in einem Fall, bei einer 65-jährigen Frau, liessen sich die Symptome - Makulaödem mit Visusstörung und periphere Ödeme - durch das Absetzen von Rosiglitazon zum Verschwinden bringen. Ein Makulaödem bei Diabeteskranken ist wohl eher selten von Rosiglitazon verursacht. Dennoch sollten Personen, die mit diesem Medikament behandelt werden, sofort ärztliche Hilfe suchen, wenn sie Sehstörungen bemerken.

Kendall C, Wooltorton E. Can Med Assoc J 2006; 174: 623

\section{Leukopenie, Thrombozytopenie}

Bei einer 50-jährigen Frau, die seit 14 Jahren wegen eines Typ-2-Diabetes Glipizid (Glibenese ${ }^{\circledR}$ ) erhielt, wurde zusätzlich Rosiglitazon verordnet. Nach 6 Monaten unter $4 \mathrm{mg}$ täglich wurde die Rosiglitazon-Dosis verdoppelt. Einige Monate später begann die Patientin über Müdigkeit und Beinmuskelkrämpfe zu klagen. Die Laboruntersuchungen ergaben eine Leukopenie $(2,500 / \mu l)$ mit einer Verminderung der Neutrophi-

\author{
Stichwortverzeichnis zu dieser Ausgabe \\ Celecoxib \\ Endometriumkarzinom (Tibolon) \\ HDL-Cholesterin, Abnahme (Rosiglitazon) \\ Herzinfarkt (Celecoxib) \\ Kreatinkinase-Anstieg (Rosiglitazon) \\ Leukopenie (Rosiglitazon) \\ Makulaödem (Rosiglitazon) \\ Mammakarzinom (Tibolon) \\ Rosiglitazon \\ Schlaganfälle (Tibolon) \\ Speicheldrüsen-Schwellung (Rosiglitazon) \\ Thrombozytopenie (Rosiglitazon) \\ Tibolon
}

\section{Texte dieser Ausgabe}

Zusammengestellt und kommentiert von E. Gysling (EG) und UP. Masche (UP) 
len und der Lymphozyten sowie eine Thrombozytopenie $\left(105^{\prime} 000 / \mu \mathrm{l}\right)$. Als Ursache der hämatologischen Anomalien wurde Glipizid vermutet. Die Leukopenie und die Thrombozytopenie blieben jedoch nach dem Absetzen des Sulfonylharnstoffs bestehen. Jetzt wurde auch das Knochenmark untersucht, wobei sich eine allgemeine Hypozellularität ergab. Rosiglitazon wurde abgesetzt und Glipizid wieder verabreicht, die Leukozyten- und Thrombozytenwerte normalisierten sich wieder.

Da der Diabetes nicht sehr gut unter Kontrolle war, wurde später doch nochmals ein Glitazon (Pioglitazon, Actos ${ }^{\mathbb{R}}$ ) verschrieben. Wieder kam es zu einer deutlichen Abnahme der Leukozyten- und der Thrombozytenzahl und wieder konnte die Situation durch Absetzen des Glitazons normalisiert werden.

Digman C et al. Ann Intern Med 2005; 143: 465-6

Bei einem 56-jährigen Arzt mit der Anamnese einer koronaren Herzkrankheit und einer Hypertonie manifestierte sich neu ein Typ-2-Diabetes. Seine Behandlung umfasste: Acetylsalicylsäure $\left(\right.$ Aspirin $^{\circledR}$ u.a.), Propranolol (Inderal ${ }^{\circledR}$ u.a.), Amlodipin (Norvasc ${ }^{\circledR}$ u.a.), Phenoxybenzamin (ein Alpharezeptorenblocker) und Simvastatin $\left(\right.$ Zocor $^{\mathbb{B}}$ u.a.). Zusätzlich wurde nun Rosiglitazon verordnet, was dosisabhängig zur Abnahme der Hämoglobin-, Leukozyten- und Thrombozytenwerte führte. Ödeme oder andere Zeichen einer Volumenüberlastung fanden sich aber nicht. Als die Rosiglitazon-Dosis von $12 \mathrm{mg}$ auf $4 \mathrm{mg}$ täglich reduziert wurde, stiegen alle Werte wieder etwas an. Aber erst ein Wechsel auf ein anderes Antidiabetikum brachte schliesslich eine markante Besserung der hämatologischen Befunde. Die Autoren des Berichtes raten zu regelmässigen Blutbildkontrollen, wenn Rosiglitazon verordnet wird.

Maaravi Y, Stessman J. Diabetes Care 2005; 28: 1536

\section{Speicheldrüsen-Schwellung}

Den kanadischen Gesundheitsbehörden liegen fünf Berichte $\mathrm{zu}$ einer Parotis-Vergrösserung unter Rosiglitazon vor. Oft handelte es sich um Kranke, die eine komplexe Anamnese aufwiesen und verschiedene Medikamente einnehmen mussten. Bei vier Personen war die Parotis beidseits angeschwollen, in einem Fall auf das Fünffache des normalen Volumens. In einem Fall war auch die Glandula submandibularis vergrössert. Die Symptomatik setzte erst 6 bis 11 Monate nach Beginn der Behandlung mit Rosiglitazon ein. Nach dem Absetzen dieses Mittels bildete sich die Schwellung mindestens in zwei Fällen wieder zurück.

http://www.hc-sc.gc.ca/dhp-mps/medeff/bulletin/carnbcei_v16n1_e.html\#5

\section{Kreatinkinase-Anstieg}

Ein 31-jähriger Mann erkrankte an einem Diabetes. Er wurde zunächst mit Metformin (Glucophage ${ }^{\circledR}$ u.a.) behandelt, nach zwei Jahren wurde Rosiglitazon hinzugefügt. Unter einer Tagesdosis von $8 \mathrm{mg}$ Rosiglitazon kam es nach zwei Monaten zu einem Anstieg der Kreatinkinase (CK) auf 746 U/1 (übliche Normwerte $<80 \mathrm{U} / \mathrm{l})$. Der Patient hatte jedoch keine klinischen Symptome. Rosiglitazon wurde abgesetzt, die CK-Werte normalisierten sich. In der Folge wurde nochmals Rosiglitazon (4 $\mathrm{mg} / \mathrm{Tag})$ gegeben und die CK-Wert stiegen innerhalb von 2 Wochen erneut an.

Sahin M et al. Diabet Med 2005; 22: 1624-5

\section{Paradoxe Abnahme der HDL-Cholesterinwerte}

Eine 61-jährige Frau mit einem Typ-2-Diabetes, diabetischer Retinopathie und Nephropathie wurde mit Fenofibrat (Lipanthyl ${ }^{\circledR}$ ), Metformin (Glucophage ${ }^{\circledR}$ u.a.), Glibenclamid (Daonil ${ }^{\circledR}$ u.a.) und Perindopril $\left(\right.$ Coversum $\left.^{\mathbb{B}}\right)$ behandelt. Drei Monate nach dem Hinzufügen von Rosiglitazon ( $8 \mathrm{mg} / \mathrm{Tag})$ war ihr HDL-Cholesterin von $1,06 \mathrm{mmol} / \mathrm{l}$ (vor Rosiglitazon) auf 0,27 $\mathrm{mmol} / \mathrm{l}$ abgesunken, die Triglyzeridwerte dagegen auf 3,4 $\mathrm{mmol} / \mathrm{l}$ angestiegen. Zwei Monate nach dem Absetzen von Rosiglitazon waren die Lipidwerte wieder normal.

Wilcox D. Can Adv React Newsl 2005; 15 (3): 2

Eine 64-jährige Frau, die wegen eines Typ-2-Diabetes während drei Jahren mit Metformin (Glucophage ${ }^{\mathbb{B}}$ u.a.) behandelt worden war, erhielt wegen weiterhin erhöhter $\mathrm{HbA}_{1 \mathrm{c}}$-Werte zusätzlich Rosiglitazon. Im Verlauf der nächsten drei Monate sank ihr HDL-Cholesterinwert von initial 1,21 $\mathrm{mmol} / 1$ auf $0,26 \mathrm{mmol} / \mathrm{l}$. Nun wurde Fenofibrat $\left(\right.$ Lipanthyl ${ }^{\circledR}$ ) hinzugefügt, was zu einem weiteren Absinken des HDL-Cholesterinwertes auf 0,11 mmol/1 führte. $\mathrm{Zu}$ diesem Zeitpunkt war auch ihr Apolipoprotein-AISpiegel sehr tief $(0,14 \mathrm{~g} / 1$ - übliche Mittelwerte um 1,5 g/l), die Triglyzeride dagegen mässig erhöht $(4,7 \mathrm{mmol} / \mathrm{l})$. Drei Monate nach Absetzen von Rosiglitazon und Fenofibrat betrug der HDL-Cholesterinspiegel 0,95 mmol/l.

Bei zwei weiteren Patienten, die ebenfalls gleichzeitig Rosiglitazon und ein Fibrat einnahmen, kam es ebenfalls zu einem starken Absinken der HDL-Cholesterin- und der Apolipoprotein-AI-Werte. Auch in diesen beiden Fällen normalisierten sich die HDL-Werte nach dem Absetzen von Rosiglitazon wieder weitgehend.

Rosiglitazon ist ein Agonist am Typ $\gamma$ des «peroxisome proliferator-activated receptor» (PPAR $\gamma$ ) während Fibrate wie z.B. Fenofibrat am PPAR $\alpha$ einwirken. Es ist nicht ausgeschlosssen, dass die paradoxe HDL-Senkung auf einer Interaktion bei gleichzeitiger Verabreichung von Stoffen aus diesen beiden Wirkstoffklassen beruht.

Sarker A et al. Diabetes Care 2004; 27: 2577-80

Dass die Glitazone keine problemlosen Medikamente sind, ist bekannt: die Entwicklung von Ödemen oder gar von einer Herzinsuffizienz ist eine Komplikation, die wir von anderen Antidiabetika nicht kennen. Schwere Leberschädigungen sind unter Pioglitazon $\left(\right.$ Actos $\left.^{\circledR}\right)$ und Rosiglitazon (Avandia ${ }^{\circledR}$ ) glücklicherweise sehr selten. Nun sind aber noch einige weitere wahrscheinlich seltene - Probleme aufgetaucht, an die man bei der Verordnung von Rosiglitazon (vielleicht auch von Pioglitazon?) denken muss. Obwohl keine klinischen Folgen des starken Absinkens der HDL-Cholesterinwerte unter Rosiglitazon + Fibrat bekannt sind, muss zweifellos geraten werden, die gleichzeitige Verabreichung dieser Medikamente nur unter Kontrolle der Lipidwerte durchzuführen.

Hier kann auch noch angemerkt werden, dass die in der Schweiz zugelassene kombinierte Behandlung mit Rosiglitazon und Insulin in Grossbritannien als kontraindiziert gilt. Eine Übersichtsarbeit kommt ebenfalls zum Schluss, die Risiken einer solchen Kombination müssten genauer untersucht werden. ${ }^{l}(E G)$

1 Scheen AJ. Drug Saf 2004; 27: 841-56 


\section{TIBOLON}

Tibolon ist ein synthetisches Steroidhormon, das in erster Linie zur Behandlung von Menopause-Symptomen zugelassen ist. Die Substanz hat neben östrogenen auch gestagene und androgene Wirkungen und führt zu einer Zunahme der Knochendichte.

Texte zu Tibolon:

Swegle JM, Kelly MW. Ann Pharmacother 2004; 38: 874-81

Gysling E. pharma-kritik 2003; 25; 17-8

Markenname: Tibolon $=$ Livial $^{\circledR}$

\section{Schlaganfälle}

In der 2001 begonnenen LIFT-Studie («Long term Intervention on Fractures with Tibolone»), wurden 4538 Frauen nach der Menopause (Durchschnittsalter: 68 Jahre) doppelblind mit Tibolon (1,25 mg/Tag) oder Placebo behandelt. Ziel der Studie war es, bei diesen Frauen, die auch eine Osteoporose aufwiesen, eine Wirkung auf die Häufigkeit von Wirbelfrakturen nachzuweisen. Die Studie wurde von einer unabhängigen Kommission («Monitoring Board») überwacht, die Zugang zu den provisorischen Resultaten hatte.

Nach durchschnittlich knapp 21/2 Jahren Studiendauer fand sich bei den Frauen, die Tibolon erhielten, eine signifikant erhöhte Inzidenz von Schlaganfällen. ${ }^{1}$ Die Studie wurde aber dennoch weitergeführt und erst rund ein halbes Jahr später abgebrochen. Bis zum Studienabbruch hatten unter Tibolon 25 Frauen $(1,11 \%)$ und unter Placebo 11 Frauen $(0,49 \%)$ einen Schlaganfall erlitten («Hazard Ratio» 2,3). Die Zahl der Wirbelfrakturen war in der Tibolongruppe signifikant (etwa um die Hälfte) kleiner als in der Placebogruppe. ${ }^{2}$

1 Grobbee DE. Br Med J 2005; 331: 843

2 Cummings SR. Br Med J 2006; 332: 667

\section{Mammakarzinom}

In Grossbritannien wurden zwischen 1996 und 2001 fast 1,1 Million Frauen im Alter zwischen 50 und 64 Jahren in die «Million Women Study» aufgenommen. ${ }^{3}$ Es handelte sich nicht um eine randomisierte Studie; die Frauen wurden befragt, ob sie Hormone einnähmen und um welche Art von Hormonen (Östrogen allein, Östrogen-Gestagen kombiniert oder Tibolon) es sich handle. Frauen, die eine Krebsanamnese hatten, wurden ausgeschlossen. Mittels einer Kennzeichnung in den Registern des britischen Gesundheitssystems konnten in der Folge die Teilnehmerinnen, die an einem Brustkrebs erkrankten oder starben, erfasst werden. Einzelheiten zur Studie lassen sich im Internet nachlesen. ${ }^{4}$

Etwa die Hälfte aller Teilnehmerinnen hatten Hormone eingenommen. Während einer Beobachtungszeit von rund 21/2 Jahren trat bei 9364 Frauen neu ein Brustkrebs auf. Da die Registrierung von Todesfällen rascher erfolgt als diejenige von Krebserkrankungen, beträgt die Beobachtungszeit bezüglich Tod infolge Brustkrebs 4 Jahre; in dieser Zeit starben 637 Frauen an Brustkrebs. Frauen, die zur Zeit des Studienbeginns oder noch kurz vorher mit Hormonen behandelt wurden, erkrankten häufiger an Brustkrebs als solche, die keine Hormone erhielten. Am höchsten war das Risiko unter einer kombinierten Östrogen-Gestagen-Therapie (relatives Risiko RR 2,0). Unter Östrogen allein betrug das RR 1,3 und unter Tibolon 1,45. Je länger die Hormonbehandlung dauerte, desto höher war das Brustkrebs-Risiko. So lässt sich schätzen, dass eine Tibolon-Behandlung während 10 Jahren zusätzlich bei 8 von 1000 Frauen zu einem Brustkrebs führt.

\footnotetext{
3 Million Women Study Collaborators. Lancet 2003; 362: 419-27

4 http://www.millionwomenstudy.org/index $2 . \mathrm{html}$
}

\section{Endometriumkarzinom}

Die oben beschriebene «Million Women Study» beschränkt sich nicht auf die Erfassung von Brustkrebs. Es sind auch Zahlen zur Inzidenz von Endometriumkarzinomen veröffentlicht. ${ }^{5}$ Diese beziehen sich auf rund 717'000 Frauen, die zur Zeit des Studienbeginns nicht hysterektomiert waren und während durchschnittlich $3 \frac{1}{2}$ Jahren beobachtet werden konnten. $45 \%$ dieser Teilnehmerinnen hatten Hormone genommen.

Das Risiko eines Endometriumkarzinoms war für Frauen unter Tibolon am grössten (relatives Risiko RR im Vergleich mit Frauen, die nie Hormone erhielten: 1,79). Das RR für Frauen unter Östrogen allein betrug 1,45. Unter einer zyklischen Östrogen-Gestagenverabreichung war das Risiko ähnlich wie bei Frauen, die keine Hormone nahmen. Dagegen war das Risiko für ein Endometriumkarzinom bei denjenigen Teilnehmerinnen, die ein Präparat mit kontinuierlicher Östrogen-Gestagenkombination nahmen, signifikant reduziert (RR 0,71).

Im Gegensatz zu den in der Veröffentlichung zum Brustkrebs ${ }^{3}$ verwendeten Daten beziehen sich die hier genannten Zahlen nicht auf «aktuell» verwendete, sondern auf die «zuletzt» verwendeten Hormonpräparate. Bei schlanken Frauen war das Risiko eines Endometriumkarzinoms allgemein grösser.

5 Million Women Study Collaborators. Lancet 2005; 365: 1543-51

Gewiss: Wie andere Hormontherapien vermag Tibolon die unangenehmen Menopause-Symptome zu reduzieren und auch einem Abbau der Knochendichte vorzubeugen. Die bis anhin oft propagierte These, Tibolon unterscheide sich vorteilhaft beispielsweise von einer kombinierten Östrogen-Gestagentherapie, hat jedoch keinen klinisch nachweisbaren Hintergrund. Im Gegenteil: es ist nicht einmal sicher, dass Tibolon nicht die riskantere Therapievariante darstellt. So fand sich das Schlaganfall-Risiko in der "Women's Health Initiative» unter kombinierter Östrogen-Gestagentherapie um 41\%, ${ }^{6}$ in der LIFT-Studie unter Tibolon aber um mehr als 100\% (gegenüber Placebo) erhöht.

Man kann nur den Kopf schütteln darüber, wie die Expertinnen und Experten immer wieder blindlings fortschrittsgläubig sind und uns ungenügend dokumentierte Medikamente als überlegene Innovationen anpreisen. (EG)

6 Writing Group for the Women's Health Initiative Investigators. JAMA 1992; 288: 321-33 


\section{CELECOXIB}

Celecoxib ist, nachdem Rofecoxib und Valdecoxib verschwunden sind, das einzige verbliebene der COX2-hemmenden «Coxibe». Der Hauptgrund, der zum Rückzug von Rofecoxib und Valdecoxib führte, lag darin, dass man vermehrte kardiovaskuläre Nebenwirkungen beobachtet hatte. Für Celecoxib wurde ebenfalls ein erhöhtes kardiovaskuläres Risiko vermutet, bislang aber nie schlüssig nachgewiesen. Die unten beschriebene Metaanalyse ändert nun dieses Bild. Informationen $\mathrm{zu}$ Celecoxib und den COX-2Hemmern:

Spanaus K, Gysling E. pharma-kritik 1998; 20: 61-4 FitzGerald GA, Patrono C. N Engl J Med 2001; 345: 433-42

Markenname: Celecoxib $=$ Celebrex $^{\circledR}$

\section{Erhöhtes Herzinfarkt-Risiko}

In einer systematischen Übersicht wurden 48 mit Celecoxib durchgeführte Doppelblindstudien identifiziert. Um daraus eine der Fragestellung gerechte Metaanalyse zusammenzustellen, war zudem gefordert, dass die Studien mindestens sechs Wochen gedauert hatten und Angaben zum Auftreten von kardiovaskulären Ereignissen geliefert worden waren. Diese Kriterien erfüllten sechs der 48 Studien - darunter auch die vielzitierte CLASS-Studie («Celecoxib Long-term Arthritis Safety Study») -, was insgesamt 12'780 Personen umfasste. Von den sechs Studien war Celecoxib in drei mit Placebo verglichen worden, in zwei mit anderen nichtsteroidalen Entzündungshemmern $\left(\right.$ Diclofenac $=$ Voltaren $^{\circledR}$ u.a., Ibuprofen $=$ Brufen $^{\circledR}$ u.a.) und in einer mit Paracetamol (Ben-u-ron ${ }^{\circledR}$ u.a.). Die verwendeten Celecoxib-Dosen lagen zwischen 200 und $800 \mathrm{mg} /$ Tag.

Für Celecoxib errechnete sich ein signifikant höheres Risiko, einen nicht-tödlichen oder tödlichen Herzinfarkt zu erleiden: im Vergleich zu Placebo allein ergab sich eine «Odds Ratio» (OR) von 2,3, im Vergleich zu Placebo und den anderen Schmerzmitteln kombiniert eine OR von 1,9. Keine signifikanten Unterschiede zwischen Celecoxib und den Kontrollgruppen fanden sich bei der Häufigkeit von zerebrovaskulären Ereignissen und kardiovaskulär bedingten Todesfällen. Will man Celecoxib nur mit anderen nicht-steroidalen Entzündungshemmern vergleichen, steht einzig die CLASS-Studie zur Verfügung; auch hier beobachtete man in der Celecoxib-Gruppe mehr Herzinfarkte als in der mit Diclofenac oder Ibuprofen behandelten Kontrollgruppe ( $\mathrm{OR}=1,7$, Unterschied allerdings nicht signifikant).

Caldwell B et al. J R Soc Med 2006; 99: 132-40

Obschon die in dieser Metaanalyse zusammengefassten Studien von der Kollektivgrösse her nicht genügend Gewicht («Power») besitzen, um das kardiovaskuläre Risiko von Celecoxib abschliessend zu analysieren, steht das Ergebnis im Einklang mit den Daten, die man mit anderen COX-2-Hemmern gesammelt hat. Man muss heute davon ausgehen, dass das erhöhte kardiovaskuläre Risiko wirklich ein Klasseneffekt der
COX-2-Hemmer ist, wahrscheinlich dadurch bedingt, dass die COX-2-Hemmung als thrombenfördernder Faktor wirkt. ${ }^{l}$ Es gibt somit keinen Grund, Celecoxib ein anderes Nutzen-RisikoVerhältnis zuzuordnen als zum Beispiel Rofecoxib.

In Neuseeland, woher die Metaanalyse stammt, hat die Herstellerfirma von Celecoxib das Ergebnis als «äusserst irreführend» abgetan. Angesichts der ganzen Geschichte der COX-2Hemmer mit zahlreichen hängigen Gerichtsverfahren deuten solche Äusserungen auch in beklemmender Weise an, dass in der Pharmaindustrie wenig Bereitschaft vorhanden ist, aus Vergangenem zu lernen. (UM)

1 Vonkeman HE et al. Br Med J 2006; 332: 895-8

\section{In den Nebenwirkungsnummern der Jahrgän- ge 25-27 (2003-05) wurden folgende Medika- mente besprochen:}

$\begin{array}{ll}\text { - Benzbromaron } & \text { - Pimecrolimus } \\ \text { - Bisphosphonate } & \text { - Rosiglitazon } \\ \text { - Celecoxib } & \text { - Sildenafil } \\ \text { - Erythromycin } & \text { - Tacrolimus } \\ \text { - Fluticason } & \text { - Tibolon } \\ \text { - Isotretinoin } & \text { - TNF-a-Hemmer } \\ \text { - Mirtazapin } & \text { - Topiramat } \\ \text { - Östrogene } & \text { - Valdecoxib } \\ \text { - Paroxetin } & \text { - Venlafaxin } \\ \text { - Pergolid } & \end{array}$

Bitte konsultieren Sie auch unsere «Bad Drug News»: http://www.infomed.org/bad-drug-news/index.php

\section{Pro memoria}

Unter dem Titel «Pro memoria» finden Sie in den pharma-kritik-Nummern dieses Jahrgangs jeweils ein Zitat aus dem Buch «100 wichtige Medikamente (2005)». Das Zitat soll zum Lesen des entsprechenden Buchkapitels anregen.

Wie lange eine auf die Symptome ausgerichtete Hormongabe dauern soll, muss in jedem einzelnen Fall mit der behandelten Frau diskutiert werden.

(Aus dem Kapitel «Estradiol», Seite 99.)

\section{pharma-kritik}

\author{
www.pharma-kritik.ch \\ e-mail: sekretariat@infomed.ch
}

Herausgegeben von Etzel Gysling (Wil)

unter Mitarbeit von Renato Galeazzi (St.Gallen) und Urs A. Meyer (Basel)

Redaktionsteam: Etzel Gysling (Leitung), Urspeter Masche, Peter Ritzmann, Thomas Weissenbach

Layout und Sekretariat: Verena Gysling

Abonnementspreis für den Jahrgang 27 (2005, 20 Nummern): 98 Franken

Erscheinungsweise: 18 Ausgaben

Infomed-Verlags-AG, Bergliweg 17, 9500 Wil

Telefon 071-910-0866, Telefax 071-910-0877

Website: www.infomed.org - e-mail: sekretariat@infomed.ch

Druck: Druckerei R.-P. Zehnder AG, 9500 Wil

(C) 2006 Infomed Wil. All rights reserved. 